\title{
On the Origin of Dynamically Cold Rings around the Milky Way
}

\section{Citation}

Younger, Joshua D., Gurtina Besla, T. J. Cox, Lars Hernquist, Brant Robertson, and Beth Willman. 2008. "On the Origin of Dynamically Cold Rings around the Milky Way." The Astrophysical Journal 676 (1): L21-24. https://doi.org/10.1086/587099.

\section{Permanent link}

http://nrs.harvard.edu/urn-3:HUL.InstRepos:41381775

\section{Terms of Use}

This article was downloaded from Harvard University's DASH repository, and is made available under the terms and conditions applicable to Open Access Policy Articles, as set forth at http:// nrs.harvard.edu/urn-3:HUL.InstRepos:dash.current.terms-of-use\#OAP

\section{Share Your Story}

The Harvard community has made this article openly available.

Please share how this access benefits you. Submit a story.

Accessibility 
Draft Version November 28, 2018

Preprint typeset using LATEX style emulateapj v. 08/22/09

\title{
ON THE ORIGIN OF DYNAMICALLY COLD RINGS AROUND THE MILKY WAY
}

\author{
Joshua D. Younger, ${ }^{1,2}$ Gurtina Besla, ${ }^{1}$ T. J. Cox, ${ }^{1,3}$ Lars Hernquist, ${ }^{1}$ Brant Robertson, ${ }^{4,5,6}$ \& Beth Willman ${ }^{1,7}$ \\ Draft version November 28, 2018
}

\begin{abstract}
We present a scenario for the production of dynamically cold rings around the Milky Way via a higheccentricity, flyby encounter. These initial conditions are more cosmologically motivated than those considered in previous works. We find that the encounters we examine generically produce a series of nearly dynamically cold ring-like features on low-eccentricity orbits that persist over timescales of $\sim 2-4 \mathrm{Gyr}$ via the tidal response of the primary galaxy to the close passage of the satellite. Moreover, they are both qualitatively and quantitatively similar to the distribution, kinematics, and stellar population of the Monoceros ring. Therefore, we find that a high eccentricity flyby by a satellite galaxy represents a cosmologically appealing scenario for forming kinematically distinct ringlike features around the Milky Way.
\end{abstract}

Subject headings: galaxy: kinematics and dynamics, galaxy: structure, galaxies: interactions, galaxies: structure, methods: $n$-body simulations

\section{INTRODUCTION}

We live in a hierarchical universe, in which mergers are a frequent occurrence (Lacey \& Cole 1993; Somerville \& Kolatt 1999; Somerville et al. 2000). In this scenario, galaxies like the Milky Way (MW) build up much of their mass by accreting smaller satellite galaxies. These minor mergers are connected to a variety of observable stellar structural and kinematic phenomena in the MW and external galaxies, including: the build-up of stellar halos (e.g. Bullock \& Johnston 2005; Bell et al. 2007), so-called "antitruncated" (Erwin et al. 2005; Pohlen \& Truiillo 2006; Younger et al. 2007) and "extended" outer disks (Ibata et al. 2005; Peñarrubia et al. 2006; Ibata et al. 2007) , and the dynamical heating of the stellar disk (e.g., Toth \& Ostriker 1992; Quinn et al. 1993; Walker et al. 1996; Kazantzidis et al. 2007).

In our own galaxy, Newberg et al. (2002) recently identified a coherent ring-like structure in Monoceros (MRi), using data from the Sloan Digital Sky Survey (SDSS: York et al. 2000). Since this initial detection, the MRi has been identified in the infrared from the Two Micron All-Sky Survey (2MASS: Skrutskie et al. 2006) by Rocha-Pinto et al. (2003) and Martin et al. (2004), reanalyzed using updated SDSS data (Grillmair 2006; Belokurov et al. 2006), and has been followed-up photometrically by several optical surveys (Ibata et al. 2003; Conn et al. 2005b, 2007). The MRi subtends $\gtrsim 100^{\circ}$ in galactic longitude and lies at a galactocentric radial distance of between 15 and $20 \mathrm{kpc}$. Spectroscopic studies have shown that it is kinematically distinct from the disk - dynamically cold with a low eccentricity orbit -

\footnotetext{
${ }^{1}$ Harvard-Smithsonian Center for Astrophysics, 60 Garden Street, Cambridge, MA 02138

2 jyounger@cfa.harvard.edu

3 Keck Foundation Fellow

${ }^{4}$ Kavli Institute for Cosmological Physics and Department of Astronomy and Astrophysics, University of Chicago, 933 East 56th Street, Chicago, IL 60637

${ }^{5}$ Enrico Fermi Insitute, 5640 South Ellis Avenue, Chicago, IL 60637

${ }_{7}^{6}$ Spitzer Fellow

${ }^{7}$ Clay Fellow
}

and is composed primarily of low-metallicity stars with $-1.6 \lesssim[\mathrm{Fe} / \mathrm{H}] \lesssim-0.4$ (Crane et al. 2003; Yanny et al. 2003; Conn et al. 2005a; Martin et al. 2005, 2006).

Numerical modeling has suggested that similar structures could be formed via tidal disruption of a low-mass satellite on a nearly coplanar orbit (Helmi et al. 2003; Meza et al. 2005; Martin et al. 2005; Peñarrubia et al. 2005, 2007). The most successful of these models argue for a very low mass companion with $M_{s} / M_{M W} \sim$ $1 / 1000$ on a nearly circular $(e=0.1)$ prograde orbit (Martin et al. 2005; Peñarrubia et al. 2005, 2007). However, recent cosmological simulations (Benson 2005; Khochfar \& Burkert 2006) have found that the orbits of satellite galaxies are likely be highly eccentric. The dynamical friction timescale for such interactions is very long and as a result it is unlikely that such a satellite could have circularized in time to form the MRi (Besla et al. 2007; Boylan-Kolchin et al. 2008). Therefore, while they are successful at reproducing many of the characteristics of the MRi, these models may have cosmologically unappealing initial conditions.

With this in mind, we propose an alternative mechanism for forming dynamically cold ring-like structures around the MW: a flyby encounter with a small satellite on a high eccentricity orbit. This scenario was first suggested by Kazantzidis et al. (2007), but here we investigate the triggering event in detail, and discuss its relevance to the $\mathrm{MRi}$.

\section{SIMULATIONS}

The simulations presented in this study were performed with GADGET2 (Springel 2005), an N-Body/SPH (Smooth Particle Hydrodynamics) code using the entropy conserving formalism of Springel \& Hernquist (2002). We include radiative cooling and star formation, tuned to fit the observed local Schmidt Law (Schmidt 1959; Kennicutt 1998). We also incorporate a subresolution multi-phase model of the interstellar medium (ISM) (Springel \& Hernquist 2003) - softened $\left(q_{E o S}=\right.$ $0.25)$ such that the mass-weighted ISM temperature is $\sim 10^{4.5} \mathrm{~K}$ - and sink particles representing supermassive black holes that accrete gas and release isotropic thermal 


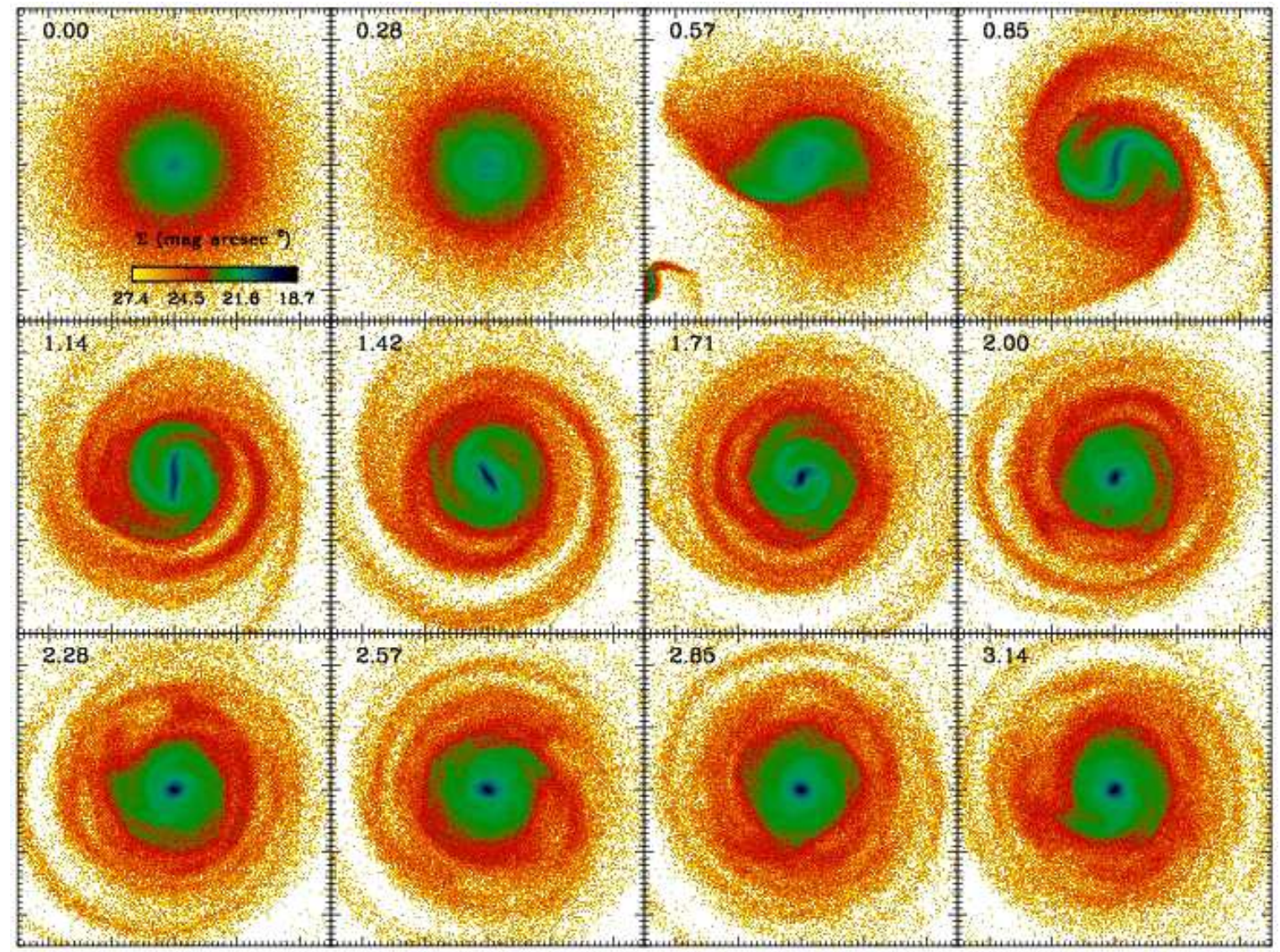

FIG. 1. - The evolution of the projected stellar mass density, colored according to a logarithmic scale. The panels are $70 \mathrm{kpc}$ on a side, and the simulation time is printed in the upper left hand corner in units of Gyr. The color-bar indicates the projected K-band surface brightness in units of mag $\operatorname{arcsec}^{-2}$, assuming a constant $M / L$ ratio of 2 in solar units.

feedback to self-regulate their growth (Springel et al. $2005 \mathrm{~b})$.

The progenitor galaxy models were constructed following Springel et al. (2005a), to which we refer the reader for details. The primary galaxy (total mass of $M_{200}=10^{12} h^{-1} M_{\odot}$ ) is analogous to the MW with a baronic mass fraction of $m_{b}=0.05$. It was realized with $10^{6}$ halo particles in a Hernquist (1990) profile with a concentration of $c=9$ as motivated by cosmological simulations (Bullock et al. 2001), and $4 \times 10^{5}$ stellar disk ( $80 \%$ of the baryonic mass) and $2 \times 10^{5}$ gas particles ( $20 \%$ of the baryonic mass). The satellite galaxy had a total mass of $M_{200}=5 \times 10^{10} h^{-1} M_{\odot}\left(M_{P G} / M_{S G} \sim 20\right)$ and an identical baryonic mass fraction. It was realized with $10^{5}$ halo particles in a Hernquist (1990) profile with a concentration of $c=18$, again motivated by cosmological simulations, and $2 \times 10^{4}$ stellar disk (50\% of the baryonic mass) and $4 \times 10^{4}$ gas particles (50\% of the baryonic mass). They were placed on a parabolic encounter with $R_{p}=5 h^{-1} \mathrm{kpc}$ perigalactic radius ( $\sim 1$ scale length $)$, consistent with the results of cosmological simulations (Benson 2005; Khochfar \& Burkert 2006).

\section{DISCUSSION}

The interaction is summarized in Figures 1 and 2. After the initial close passage, resonances between the orbital frequency of the satellite and primary galaxy disk particles ("stars") excite coplanar tidal arms (Toomre \& Toomre 1972). These features then wrap around the primary galaxy as it continues to revolve, forming a set of concentric ring-like features. Star formation induced by the merger is concentrated in the nucleus of the primary galaxy (see e.g., Hernquist 1989; Mihos \& Hernquist 1994, 1996; Hernquist \& Mihos 1995), making these rings primarily disk stars. They are furthermore dynamically cold and kinematically distinct from typical disk stars (see Figure 3), and slowly disperse on a timescale of $\sim 2-4 \mathrm{Gyr}$ (or several rotation periods) owing to phase mixing of the collisionless stellar particles (Binney \& Tremaine 1987). Because these rings are generated via gravitational interactions, they are largely insensitive to the gas content or structural parameters of the satellite galaxy. And because the flyby is effectively an impulse interaction, they will still be formed even if the satellite is disrupted during the encounter.

\section{RELEVANCE TO THE MONOCEROS RING}

The ring features that are produced by the flyby interaction are similar in several ways to the MRi first identified by Newberg et al. (2002). They provide a good match to the kinematics and location of the ring, and are roughly consistent with metallically measurements of MRi stars. And, cosmological simulations indicate that such interactions are common for MW-sized halos over the timescale for ring formation (i.e., within the past $\sim 4$ Gyr; Stewart et al. 2007). Therefore, while we do not claim to have modeled the MRi in detail, based on these similarities we propose a flyby interaction as a possible formation scenario. 

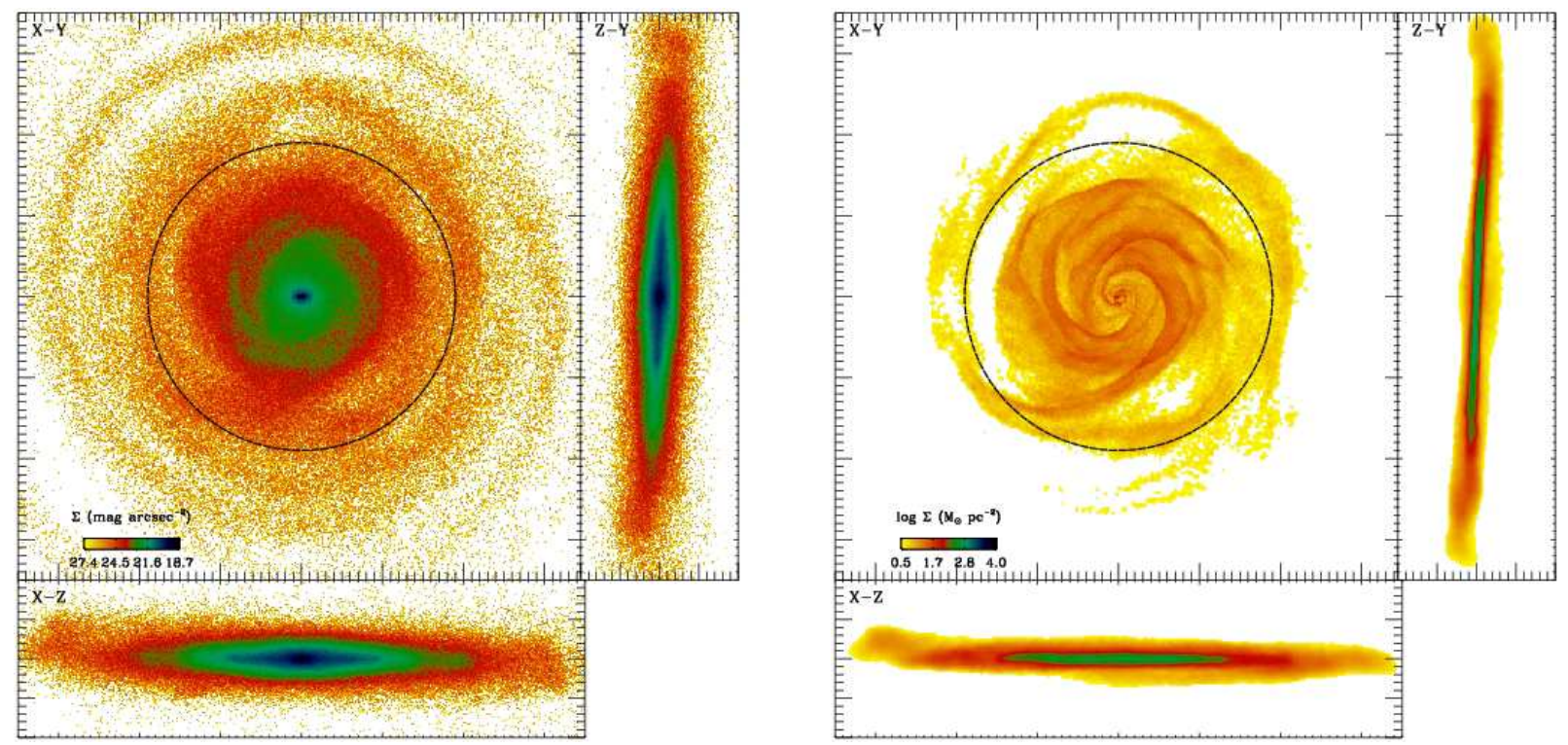

FIG. 2.- Three different projections (X-Y, Z-Y, and X-Z) of the K-band surface brightness (left; again assuming a constant $M / L$ ratio of 2 in solar units) and gas surface mass density (right) at $t \approx 3$ Gyr. Panels are $50 \mathrm{kpc}$ on a side in the $\mathrm{X}$ and $\mathrm{Y}$ directions, and $20 \mathrm{kpc}$ in the $\mathrm{Z}$ direction. The solid line corresponds to one of the ring structures as roughly the same galactocentric location $\left(R_{g a c}=19 \mathrm{kpc}\right)$ as the MRi.
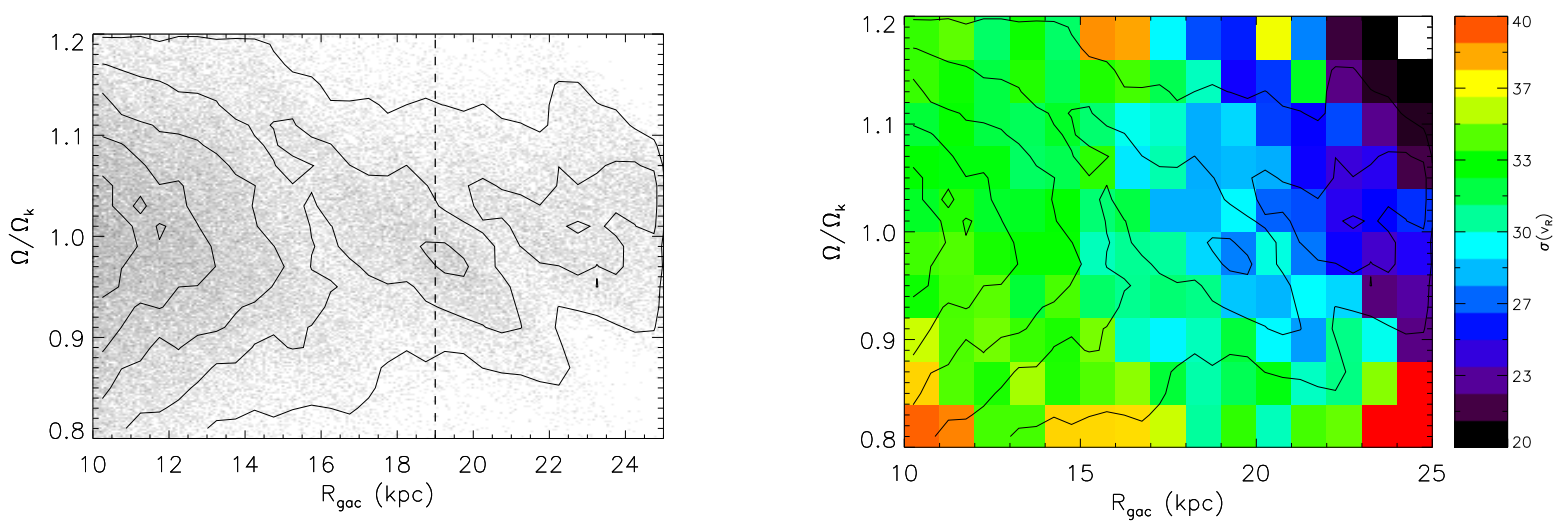

FIG. 3.- Phase space diagram for the stellar particles at $t \approx 3$ Gyr, showing the orbital frequency $(\Omega)$ as a fraction of that expected from a circular orbit at that radius $\left(\Omega_{k}\right)$ as a function of galactocentric radius $\left(R_{\text {gac }}\right)$. The left panel shows the density of points in gray-scale, with linear contours overlaid to guide the eye and a dashed line at the location indicated in Figure 2 The right hand panel is color coded by the radial velocity dispersion $\left(\sigma\left(v_{R}\right)\right)$ in each cell, with the same contours overlaid.

The MRi forms a coherent structure over $100^{\circ}$ in galactic longitude from approximately $15-20$ kpc from the galactic center (Newberg et al. 2002; Ibata et al. 2003; Rocha-Pinto et al. 2003; Martin et al. 2004; Conn et al. 2005b, 2007). Kinematically, it is distinguished from MW disk stars by its low radial velocity dispersion $\left(\sim 25 \mathrm{~km} \mathrm{~s}^{-1}\right)$; the MRi is dynamically cold (Crane et al. 2003; Yanny et al. 2003; Conn et al. 2005a; Martin et al. 2005, 2006). Proper motion measurements ${ }^{8}$ also suggest that the ring stars are in low-eccentricity orbits (e.g., Crane et al. 2003; Yanny et al.|2003). We find

\footnotetext{
8 Crane et al. (2003) measure an overall circular velocity of $\sim 100 \mathrm{~km} \mathrm{~s}^{-1}$. However, we note (as in Peñarrubia et al. 2005) that the accuracy of such measurements in physical units is limited to $\Delta v_{\text {perp }} \approx 4.74 R_{\odot} \Delta \mu$ where $R_{\odot} \approx 8 \mathrm{kpc}$ is the galactocentric radius of the sun and $\Delta \mu \sim 3-4$ mas $\mathrm{yr}^{-1}$ is the typical proper motion measurement error. We have therefore chosen to interpret these measurements as being generally consistent with low-eccentricity orbits.
}

that our simulations produce a ring in the right galactocentric radial range that is approximately circularly supported $-0.9 \lesssim \Omega / \Omega_{k} \lesssim 1.1$, where $\Omega$ is the orbital frequency and $\Omega_{k}$ is that expected from circular motion - with a similarly low radial velocity dispersion (see Figure 3). This ring also extends $-4 \lesssim z \lesssim 4 \mathrm{kpc}$ above and below the galactic plane, which is consistent with detections such as those of Conn et al. (2007). Although it is unclear what fraction of the ring would be identified in observations, it contains $\lesssim 1 \%\left(\lesssim 5 \times 10^{8} M_{\odot}\right)$ of the total stellar mass of the disk, which is consistent with the mass estimates of Yanny et al. (2003).

A disk origin for MRi stars is also broadly consistent with their observed stellar population. Several spectroscopic studies (e.g., Yanny et al. 2003) have found mean metallicities of $[\mathrm{Fe} / \mathrm{H}]=-1.6 \pm 0.3$, while Crane et al. (2003) find a higher mean metallicity of $[\mathrm{Fe} / \mathrm{H}] \approx-0.4$ using a different tracer population. This suggests a primarily metal-poor stellar population with a spread in 


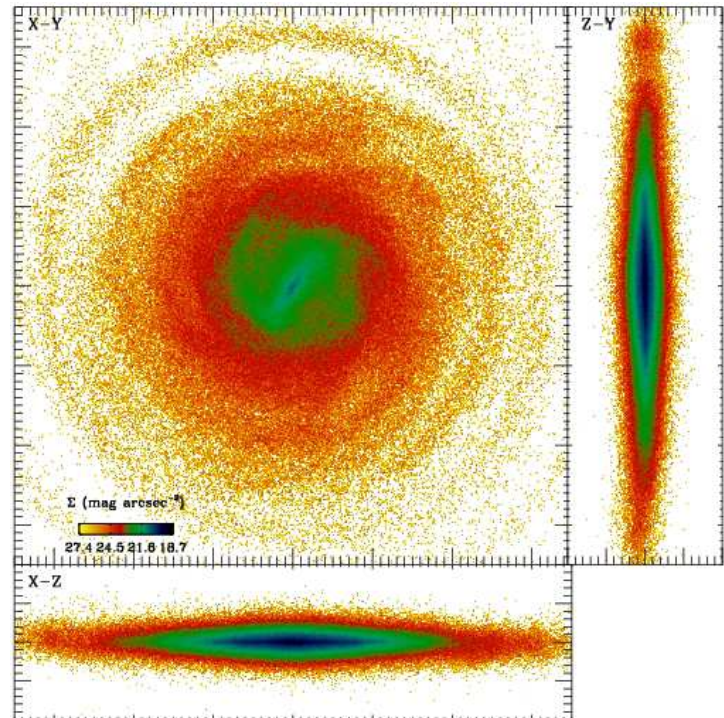

FIG. 4.- Three projections (X-Y, Z-Y, and X-Z) of the K-band surface brightness (again assuming a constant $M / L$ ratio of 2 in solar units) at the same simulation time as Figures 2 and 3 for a $M_{S G} / M_{P G} \approx 1 / 100$ interaction with identical orbital parameters.

metallicities, and possibly multiple epochs of star formation. Our modeling indicates that the MRi may have formed from outer disk stars moved outwards by tidal interactions with the satellite galaxy, which is consistent with this metal-poor stellar population (Luck et al. 2006; Yong et al. 2006). Furthermore, the tidal interaction moves a significant supply of cold gas into the same ring structures (see Figure 2), which provides the raw material for subsequent epochs of star formation. While the density-dependent prescription in our simulations does not accurately capture some modes of star formation owing to the limitations of the SPH method, prescriptions that include an approximate treatment of shocks (e.g., Barnes 2004) may show these multiple star formation episodes.

While ring features in our simulations capture many of the properties of the MRi, there are some observations which potentially conflict with our proposal. In particular, the satellite in our simulations is roughly the same mass as the LMC, and by $\sim 2$ Gyr after the interaction would be at a galactocentric distance of $\sim 250$ kpc. Such a massive object is well within the detection threshold of current surveys (e.g., Willman et al. 2002; Koposov et al. 2007); Leo I - a far lower mass MW companion - was detected at comparable distance (Caputo et al. 1999; Bellazzini et al. 2004; Mateo et al. 2007). However, dynamically cold ring structures are produced generically in flyby encounters, and similar features are present in both a lower-inclination interaction $\left(i=10^{\circ}\right)$ and for a lower mass satellite galaxy $\left(M_{S G} / M_{P G} \sim 1 / 100\right.$; Figure 4). It is thus possible that

9 In our simulations, both the $1 / 20$ and $1 / 100$ encounters stripped $\sim 50 \%$ of the satellite mass. While the efficiency of this process is sensitive to both the initial structure of the satellite and the orbital parameters of the interaction, this suggests that a a the satellite galaxy that produced the MRi is either (1) hidden from view, at low galactic latitude and/or on the other side of the galaxy, or (2) it is lower mass with $1 / 100 \lesssim M_{S G} / M_{P G} \lesssim 1 / 20$. Therefore, it is not unreasonable to speculate that Leo I, which had at least one passage through the MW disk within the past 2-4 Gyr (Sohn et al. 2007), could have excited the MRi we see today. Moreover, it is likely that this interaction stripped a significant fraction of its mass $^{9}$, making current dynamical estimates $\left(M_{\text {Leo I }} / M_{M W} \sim 1 / 1000\right.$; Mateo et al. 2007) a lower limit on its mass at the time of the interaction. Furthermore, simulations indicate that there are likely $\gtrsim$ several sub-haloes in the MW halo with $1 / 100 \lesssim M_{S H} / M_{P G} \lesssim 1 / 20$ on orbits that take then through the disk (Moore et al. 1999; Giocoli et al. 2007; Kazantzidis et al. 2007). Since galaxy formation may be inefficient in low mass haloes (e.g., Haiman et al. 1997; Barkana \& Loeb 1999; Kravtsov et al. 2004), they may be significantly underluminous and therefore difficult to identify.

Finally, there have been suggestions in the literature (Crane et al. 2003; Frinchaboy et al. 2004) that some globular clusters (GCs) may be associated with the MRi. This is also potentially in conflict with our modeling. However, we note that their physical association with the MRi is somewhat speculative. Alternatively, there are $\sim$ several nearly coplanar GCs at roughly the same radius (Harris 1996) as progenitor MRi stars in our simulations that they could have also been moved into the rings by the interaction.

\section{CONCLUSION}

We present a scenario for the production of dynamically cold rings around a disk galaxy such as the MW via a prograde flyby encounter with a satellite galaxy. Tidal arms excited during close passage coalesce and wrap around the disk of the primary galaxy. These kinds of interactions are more cosmologically likely than the nearly circular orbits presented by Martin et al. (2005) and Peñarrubia et al. (2005, 2007), while dynamical friction is insufficient to circularize the orbit of such a low mass companion. Our modeling shows rings with similar spatial distribution and kinematics to the MRi. The disk origin of MRi stars is furthermore broadly consistent with observed stellar populations. Therefore, we find that a flyby encounter represents a more cosmologically appealing scenario for the production of the MRi and other dynamically cold rings around the MW.

Thanks to the anonymous referee for helpful comments, and to M. Ashby, J. Bullock, S. Dutta, P.. F. Hopkins, C. Hayward, and S. Bush for valuable discussions. This work was supported in part by a grant from the W. M. Keck foundation.

passage through the disk is likely to remove a significant fraction of the satellite's total mass.

\section{REFERENCES}

Barkana, R. \& Loeb, A. 1999, ApJ, 523, 54

Barnes, J. E. 2004, MNRAS, 350, 798
Bell, E. F. et al. 2007, ApJ, submitted [astro-ph/0706.0004] Bellazzini, M. et al. 2004, MNRAS, 354, 708 
Belokurov, V. et al. 2006, ApJ, 642, L137

Benson, A. J. 2005, MNRAS, 358, 551

Besla, G. et al. 2007, ApJ, 668, 949

Binney, J. \& Tremaine, S. 1987, Galactic dynamics (Princeton, NJ, Princeton University Press, 1987, 747 p.)

Boylan-Kolchin, M., Ma, C.-P., \& Quataert, E. 2008, MNRAS, 383, 93

Bullock, J. S. \& Johnston, K. V. 2005, ApJ, 635, 931

Bullock, J. S. et al. 2001, MNRAS, 321, 559

Caputo, F. et al. 1999, AJ, 117, 2199

Conn, B. C. et al. 2005a, MNRAS, 364, L13

-. 2005b, MNRAS, 362, 475

-. 2007, MNRAS, 376, 939

Crane, J. D. et al. 2003, ApJ, 594, L119

Erwin, P., Beckman, J. E., \& Pohlen, M. 2005, ApJ, 626, L81

Frinchaboy, P. M. et al. 2004, ApJ, 602, L21

Giocoli, C., Tormen, G., \& van den Bosch, F. C. 2007, MNRAS, submitted [astro-ph/0712.1563]

Grillmair, C. J. 2006, ApJ, 651, L29

Haiman, Z., Rees, M. J., \& Loeb, A. 1997, ApJ, 484, 985

Harris, W. E. 1996, AJ, 112, 1487

Helmi, A. et al. 2003, ApJ, 592, L25

Hernquist, L. 1989, Nature, 340, 687

-. 1990, ApJ, 356, 359

Hernquist, L. \& Mihos, J. C. 1995, ApJ, 448, 41

Ibata, R. et al. 2005, ApJ, 634, 287

-. 2007, astro-ph/0704.1318

Ibata, R. A. et al. 2003, MNRAS, 340, L21

Kazantzidis, S. et al. 2007, ApJ, submitted [astro-ph/0708.1949]

Kennicutt, Jr., R. C. 1998, ApJ, 498, 541

Khochfar, S. \& Burkert, A. 2006, A\&A, 445, 403

Koposov, S. et al. 2007, ApJ, submitted [astro-ph/0706.2687], 706

Kravtsov, A. V., Gnedin, O. Y., \& Klypin, A. A. 2004, ApJ, 609, 482

Lacey, C. \& Cole, S. 1993, MNRAS, 262, 627

Luck, R. E., Kovtyukh, V. V., \& Andrievsky, S. M. 2006, AJ, 132,902

Martin, N. F. et al. 2004, MNRAS, 348, 12

—. 2005, MNRAS, 362, 906
—. 2006, MNRAS, 367, L69

Mateo, M., Olszewski, E. W., \& Walker, M. G. 2007, ApJ, in press [astro-ph/0708.1327]

Meza, A. et al. 2005, MNRAS, 359, 93

Mihos, J. C. \& Hernquist, L. 1994, ApJ, 431, L9

-. 1996, ApJ, 464, 641

Moore, B. et al. 1999, ApJ, 524, L19

Newberg, H. J. et al. 2002, ApJ, 569, 245

Peñarrubia, J., McConnachie, A., \& Babul, A. 2006, ApJ, 650, L33

Peñarrubia, J. et al. 2005, ApJ, 626, 128

—. 2007, ApJL, submitted [astro-ph/0703601]

Pohlen, M. \& Trujillo, I. 2006, A\&A, 454, 759

Quinn, P. J., Hernquist, L., \& Fullagar, D. P. 1993, ApJ, 403, 74

Rocha-Pinto, H. J. et al. 2003, ApJ, 594, L115

Schmidt, M. 1959, ApJ, 129, 243

Skrutskie, M. F. et al. 2006, AJ, 131, 1163

Sohn, S. T. et al. 2007, ApJ, 663, 960

Somerville, R. S. \& Kolatt, T. S. 1999, MNRAS, 305, 1

Somerville, R. S., Lemson, G., Kolatt, T. S., \& Dekel, A. 2000, MNRAS, 316, 479

Springel, V. 2005, MNRAS, 364, 1105

Springel, V., Di Matteo, T., \& Hernquist, L. 2005a, ApJ, 620, L79

-. 2005b, MNRAS, 361, 776

Springel, V. \& Hernquist, L. 2002, MNRAS, 333, 649

-. 2003, MNRAS, 339, 289

Stewart, K. R. et al. 2007, ApJ, submitted [astro-ph/0711.5027], 711

Toomre, A. \& Toomre, J. 1972, ApJ, 178, 623

Toth, G. \& Ostriker, J. P. 1992, ApJ, 389, 5

Walker, I. R., Mihos, J. C., \& Hernquist, L. 1996, ApJ, 460, 121

Willman, B. et al. 2002, AJ, 123, 848

Yanny, B. et al. 2003, ApJ, 588, 824

Yong, D. et al. 2006, AJ, 131, 2256

York, D. G. et al. 2000, AJ, 120, 1579

Younger, J. D., Cox, T. J., Seth, A. C., \& Hernquist, L. 2007, ApJ, 670, 269 